\title{
Standardization of nucleic acid amplification tests in diagnostic molecular microbiology
}

\author{
Alberta Azzi, Federico Chiodo, Marina Crovatto, Valeria Ghisetti, Carlo Mancini, \\ Paolo Ravanini, Marisa Zerbini \\ Comitato di Studio per la Biologia Molecolare (CoSBioM)* dell'AMCLI
}

Key words: Standardization, Quality control, Multicentre studies, Real time PCR

\section{SUMMARY}

Nucleic acid detection by amplification (NAT) assays began to be used in diagnostic microbiology about twenty years ago. Since then, the progress of molecular methods has been continuous and rapid, aimed to obtain more and more sensitive and specific results and automation. To actually achieve such objectives, microbiology laboratories have to use suited quality controls and to participate to external quality control programs. Quality controls for NAT assays need to be adjusted to follow the evolution of the molecular methods. The need of standardization and suited controls for NAT assays became evident approximately by the middle of ' 90 years. Thus International Standards (IS) were established by the World Health Organization, for the main blood borne viruses. Such preparations had a well known concentration expressed as International Units, a new measure unit for nucleic acid quantitation. Then, IS were used in order to calibrate working reagents and synthetic calibrators. Another tool for standardization are multicentre studies aimed to verify the performances of participating laboratories using different molecular methods, and allow the comparison of inter-laboratory results. The CoSBio of AMCLI, as other European organizations, in the last years promoted, some multicentre studies on NAT assays. The results of such studies demonstrated a good level of diagnostic performances of participating laboratories. However, with the introduction of the real time PCR (RQ-PCR) a new problem emerged: the variability of results obtained by molecular methods due to different coefficient of linear amplification. Solutions suggested by Literature to this and similar problems are examined, mainly focusing on the assessment of the Calibration curves in the RQ-PCR. Finally, the Authors signal some important fields in the molecular diagnostics, such as the detection of HPV and the detection of BKV, still lacking of sufficient standardization.

\section{Received September 28, 2007}

Controllo di qualità nel laboratorio diagnostico Definizioni

Controllo di qualità (quality control)

Con il termine controllo di qualità ci si riferisce a quelle misure che devono essere incluse durante ciascuna seduta di laboratorio per verificare che il test stia funzionando adeguatamente.

Assicurazione della qualità (quality assurance) definisce quell'insieme di procedure che assicurano che $\mathrm{i}$ risultati finali riportati dal laboratorio sono corretti.

Scopo del controllo di qualità è quello di assicurare che i risultati generati dal test siano corretti; comunque l'assicurazione della qualità deve essere molto più ampia, prevedendo anche il controllo dell'accuratezza della fase pre- e post-analitica: che il test giusto sia eseguito sul campione giusto e che il giusto risultato e la giusta interpretazione sia inviata alla persona giusta nel tempo giusto.
Accepted November 5, 2007

Gestione della qualità (Quality assessment).

La gestione della qualità, nota anche come test di efficacia, è uno strumento per determinare la qualità dei risultati generati dal laboratorio.

La gestione della qualità è una verifica dell'efficacia dell'assicurazione di qualità e del controllo di qualità. La gestione della qualità può includere valutazione interne o esterne.

Variabili che possono alterare la qualità dei risultati.

1. Formazione e aggiornamento del personale del laboratorio;

2. le condizioni dei campioni da esaminare;

3. i controlli usati in ogni corsa;

4. i reagenti;

5. la strumentazione;

6. l'interpretazione dei risultati;

7. la refertazione (trascrizione e presentazione dei risultati). 


\section{INTRODUZIONE}

La ricerca degli acidi nucleici, soprattutto tramite tecniche di amplificazione di sequenze geniche bersaglio, si è affermata in poco più di un ventennio in diversi settori della diagnostica microbiologica, incrementandone significativamente le potenzialità.

L'origine di questa rivoluzione nella diagnostica microbiologica si fa risalire alla scoperta di una DNA polimerasi termostabile (Kary Banks Mullis, 1983); infatti l'uso di questo enzima consentì lo sviluppo e l'ampia applicazione della reazione polimerasica a catena (PCR), che è diventata la più utilizzata reazione di amplificazione del DNA.

Già nel 1985 Saiki (24) pubblicava una prima applicazione della PCR per la diagnosi molecolare dell'anemia falciforme.

Nel 1988 furono pubblicate le prime applicazioni della PCR alla diagnostica virologica per la ricerca di HIV(17) e di HHV6 (2).

Nel 1992 furono introdotti i primi kit commerciali per la diagnosi di infezione da C. trachomatis e da HIV.

La tecnica di PCR è stata poi sviluppata in "formati” diversi, quali la PCR nested e la PCR multiplex, allo scopo di aumentarne la sensibilità, la specificità e di adattarla a esigenze diagnostiche di tipo diverso. Alla PCR si affiancarono negli anni successivi altre tecniche di amplificazione che contribuirono al grande sviluppo della diagnostica microbiologica e soprattutto di quella virologica. Tra le cosiddette tecniche di amplificazione del bersaglio, oltre alla PCR, si è affermata la Nucleic Acid Sequenze-Based Amplification (4) (NASBA), la Transcription-Mediated Amplification (TMA) (10) e la Strand Displacement Amplification (SDA) (31). Molte sono le applicazioni diagnostiche per cui sono state proposte tali tecniche e uno dei loro vantaggi, in contrapposizione alla PCR, è l'essere basate su reazioni isoterme e quindi non dipendenti dalla disponibilità di thermal cycler, strumento necessario invece per la PCR. I thermal cycler, soprattutto nei primi anni dopo l'introduzione della PCR, erano strumenti abbastanza costosi e da aggiungere al già lungo elenco di strumenti di laboratorio da "controllare" rigorosamente nelle pratiche di controllo di qualità interno dei laboratori. È ben noto ed evidente, infatti, che variazioni nell'efficienza dei thermal cycler, nella velocità con cui si effettuano i cambiamenti di temperatura, la non perfetta uniformità delle temperature nei pozzetti possono influire in maniera non lieve sui risultati delle reazioni.

In aggiunta e in alternativa alla PCR che si basa sull'amplificazione genica del bersaglio, hanno trovato impiego diagnostico anche reazioni di amplificazione di sequenze geniche di rilevamen- to (sonde molecolari), quali la ligase chain reaction (LCR) (13). Hanno inoltre dimostrato grande validità anche alcune reazioni di ibridazione basate sull'amplificazione del segnale, quali le tecniche branched DNA (bDNA)(29) e hybrid capture (HC) (27).

Le tecniche di amplificazione genica, la PCR in particolare, oltre ad avere uno scopo diagnostico qualitativo, trovano anche una applicazione quantitativa. Nel settore diagnostico microbiologico le tecniche di amplificazione qualitative e quantitative trovano applicazione in quei settori in cui la ricerca del patogeno è impossibile mediante i metodi colturali tradizionali oppure richiederebbe dei tempi particolarmente lunghi di coltivazione, non adeguati ad uno scopo diagnostico.

Alla necessità di individuazione del o dei patogeni con saggi qualitativi, si è ormai affiancata l'esigenza di tecniche quantitative per monitorare la carica infettante, in particolare in corso di terapia o in corso di infezioni croniche nel paziente immunodepresso. Alle metodiche molecolari quantitative basate sulla tecnica di PCR competitiva end-point, si stanno sostituendo rapidamente le tecniche di PCR real time (RQ-PRC). Le RQPCR si basano sul monitoraggio costante e continuo della reazione di amplificazione mediante incorporazione di un fluorocromo intercalante o mediante il rilevamento di un segnale emesso da una sonda marcata.

I saggi di amplificazione genica che vengono utilizzati nel laboratorio diagnostico devono essere accuratamente standardizzati e validati. La standardizzazione può essere ottenuta con l'utilizzo di adeguati controlli interni (run controls), possibilmente calibrati su standard internazionali. L'uso di standard internazionali garantisce l'omogeneità dei risultati tra diversi laboratori e tra diversi saggi diagnostici. Prima di validare un metodo quindi, i laboratori devono ottimizzarlo e documentare il protocollo. Il processo di validazione include una serie di fasi:

1. valutazione di una serie di diluizioni di campioni positivi per determinare il limite di sensibilità del saggio e, nei test quantitativi, deve essere dimostrata la linearità delle concentrazioni testate;

2. determinazione del coefficiente di variazione del saggio nell'ambito di diverse sedute;

3. la determinazione della sensibilità e la specificità e il grado di cross-reattività con altro materiale genomico;

4. si deve assicurare la qualità dell'intero saggio utilizzando procedure di controllo di qualità che siano in grado di monitorare il comportamento di differenti lotti di reagenti prima che un nuovo lotto venga introdotto nel saggio. 
Il principale limite ad un più ampio impiego delle tecniche molecolari è il loro costo elevato.

Una ragione per cui i saggi commerciali sono ancora molto costosi è anche la necessità di una estesa validazione prima che il kit sia immesso sul mercato poiché i produttori devono garantire l'affidabilità della performance dei loro saggi, assumendo che le istruzioni vengano seguite correttamente.

Come si vede nella tabella 1 (33), riportata a titolo indicativo, molte di queste tecniche si sono tradotte in saggi commerciali che sono stati opportunamente validati e quindi approvati dall'FDA.

È possibile consultare un elenco aggiornato al 2007 dei test approvati dall'FDA all'indirizzo: http://www.amp.org/FDATable/FDATableMar07. doc.

La grande variabilità delle tecniche molecolari disponibili, a partire dalle fasi di pretrattamento dei campioni per l'estrazione degli acidi nucleici fino alle fasi di rivelazione dei prodotti della reazione, la possibilità di individuare uno stesso patogeno mediante la ricerca di sequenze diverse e la varietà della strumentazione utilizzabile, fino alla completa automatizzazione delle procedure, rendono indispensabile nel laboratorio diagnostico un efficiente controllo di qualità.
Gli strumenti della standardizzazione:

Standard, controlli, EQC (external quality control)

L'esigenza prioritaria di un efficiente controllo di qualità fu messa in luce ed efficacemente dimostrata in indagini multicentriche dal confronto dei risultati dell'analisi di una stessa serie di campioni codificati, ottenuti dai diversi laboratori partecipanti. Uno dei primi studi di questo tipo è quello di Zaaijer nel 1993 (34), sulla standardizzazione della PCR nella diagnosi di infezione da HCV. Esso dimostrò che solo il 16\% dei laboratori partecipanti non aveva commesso errori analizzando un pannello di sieri HCV negativi e positivi a varie concentrazioni.

Negli anni successivi, altri studi analoghi furono condotti per la standardizzazione dei metodi amplificazione molecolare (NAT), particolarmente per la ricerca di alcuni patogeni tra cui $\mathrm{HCV}$ e HIV, di largo impiego in campo trasfusionale. Questi studi misero in evidenza la necessità di avere a disposizione dei campioni di riferimento, internazionalmente riconosciuti, che permettessero di calibrare i diversi metodi di amplificazione disponibili, al fine di ottenere risultati sovrapponibili e ripetibili tra i vari laboratori. Nasce così il concetto di Standard Internazionale (SI) (tabella 2) e di Unità Internazionale (UI), in sostituzione

Tabella I. FDA-approved nucleic-acid-based assays for detection of microbial pathogens.

\begin{tabular}{|c|c|c|c|c|c|}
\hline Organism detected & Trade name & Company/institution & Method & Clinical sensitivity & Clinical specificity \\
\hline \multirow{6}{*}{ Chlamydia trachomatis } & Amploor & Roche & PCR & $93 \cdot 2 *$ & $98.41^{10}$ \\
\hline & LCX & Abbott & LCR & $>95^{10}$ & $>99:$ \\
\hline & AMP & Gen-Probe & TMA & $86 \cdot 7-99 \cdot 2^{\circ}$ & $>99^{\prime 2}$ \\
\hline & PACE 2 & Gen-Probe & Hybridisation & $608-78 \cdot 1^{36}$ & $>99^{16}$ \\
\hline & BCFrobeTec & Becton Dickinson & SDA & $94 \cdot 0^{\circ 5}$ & $>99 *$ \\
\hline & Hybrid capture II CT-ID & Digene & Hybrid capture & $95 \cdot 4^{3 \pi}$ & gent \\
\hline \multirow[t]{2}{*}{ Cytomegalosinus } & CIN pp 67 mRNA & Organon Teknika & NASBA & $95^{*}$ & $98^{2}$ \\
\hline & Hybrid capture CMV CNA test & Digene & Hybrid capture & $95^{10}$ & $96^{23}$ \\
\hline Gorcherella vaginal's & Affim VP III & Becton Dickinson & Hybridisation & $94^{42}$ & $81^{20}$ \\
\hline Group A streptococas & GP-ST teet & Gen-Probe & Hybridisation & $88 \cdot 6^{\prime \prime}$ & $978^{10}$ \\
\hline Group B streptococcus & IDIStrepB & Irfectio Diegnostics & Real-time PCR & $97^{\prime 9}$ & $100^{\circ}$ \\
\hline \multirow{3}{*}{$\mathrm{HCV}$} & Ampicor HCV & Roche & PCR & $98^{13}$ & NA \\
\hline & Versant HCV RNA qualtative assey & Bayer & TMA & NA & $96^{\prime \prime}$ \\
\hline & Versant HCV RNA 3.0 & Bayer & BCNA & NA & $98 \cdot 2^{\circ}$ \\
\hline \multirow[t]{6}{*}{$\mathrm{HN}$} & Amploor HN-1 Moritor Test & Roche & RT-PCR & NA & $>99^{\prime \prime}$ \\
\hline & $\begin{array}{l}\text { Trugene HVV drug resistance and } \\
\text { OperKene DNA sequencing }\end{array}$ & Visble Genetics & CNA sequencing & NA & NA \\
\hline & NudíSens Easya HIV-1 & bicMsrieux & NASBA & NA & $>99: 3$ \\
\hline & Procleix $\mathrm{HN}-1 / \mathrm{HCV}$ & Chiron & TMA & $>99^{a}$ & $>99^{20}$ \\
\hline & Versant HN-1 RNA 3.0 & Bayer & BCNA & & $97 \cdot 6^{u}$ \\
\hline & Viroseq & Applied Biosystems & DNA sequencing & NA & NA \\
\hline \multirow{3}{*}{$\begin{array}{l}\text { HFV } \\
\text { M tuberculosis }\end{array}$} & Hybrid capture II HPV DNA & Digene & Hybrid capture & $>99^{i}$ & $85-90^{46}$ \\
\hline & TB Amplicor & Roche & PCR & $79 \cdot 4-91 \cdot 9^{\circ}$ & $>99^{\prime 2}$ \\
\hline & E-MTD & Gen-Probe & TMA & $90 \cdot 9-95 \cdot 2^{10}$ & $>99^{\prime \prime}$ \\
\hline \multirow[t]{5}{*}{ Neisseria gonomhoeae } & Amplioor & Roche & PCR & NA & NA \\
\hline & LCX & Abbott & LCR & $>95^{10}$ & $>99: 8$ \\
\hline & Hybrid capture \| CT/GC & Digene & Hybrid capture & $90^{45}$ & $98 \cdot 5^{5}$ \\
\hline & BCProbe Tec & Becton Dickineon & SDA & $88 \cdot 95$ & $>99 *$ \\
\hline & PACE-2 & Gen-Probe & Hybridisation & $97^{16}$ & $9 \theta^{\circ}$ \\
\hline Trichomanes vaginal's & Affim VP III & Becton Dickinson & Hybridisation & $88-91 g^{2}$ & $100^{\circ}$ \\
\hline
\end{tabular}

Tratta da Yang e Rothman (33) 
delle varie unità utilizzate per esprimere la concentrazione delle sequenze di DNA/RNA bersaglio (numero di copie di genomi per ml o genomi equivalenti per $\mathrm{ml}$; unità di PCR; pg di DNA/RNA ...).

Il primo SI, approvato dall'Organizzazione Mondiale della Sanità (WHO) nel 1997 è stato quello per $\mathrm{HCV}$, frutto di uno studio collaborativo, poi pubblicato nel 1999 (25). Questo studio multicentrico servì per scegliere, tra le 3 proposte, la preparazione più adatta a rappresentare l'SI e per definirne la concentrazione in UI. In base al titolo medio della preparazione scelta, ottenuto in saggi a diluizione limite dai diversi laboratori partecipanti, la concentrazione assegnata a questa preparazione fu di $10^{5} \mathrm{UI} / \mathrm{ml}$. I dati e le indicazioni fornite dallo studio furono poi valutati da parte del Comitato di Esperti del WHO per la Standardizzazione Biologica per la definitiva accettazione. Quel primo SI per HCV RNA (tabella 2) venne codificato come NIBS 96/790 e consisteva in una preparazione liofilizzata di $\mathrm{HCV}$, di genotipo 1, diluito in un criosovranatante HCV-negativo.

In modo analogo, negli anni immediatamente suc-

Tabella 2. Definizione degli standard internazionali secondo WHO

\section{WHO International Standards}

- Sono la base per la calibrazione delle curve di riferimento di tutti i metodi per la diagnostica molecolare.

- Vengono espressi in IU/ml per evitare le variabili dovute all'utilizzo di differenti unità di misura quali copie $/ \mathrm{ml}$ o mEq/ml.

- Sono selezionati in studi policentrici, ove vengono utilizzati metodi analitici con specifiche caratteristiche (Coefficienti di Amplificazione Lineare-CAL).

Per il I WHO I. S. 2001/2005 (96/970-96/978) dell'HCV-RNA sono stati utilizzati:

Roche-Amplicor
Chiron-Quantiplex
Organon Teknica NASBA and
House-assays(single or nested PCR)

Vox Sang 1999/2001 Saldanha J. zione lineare (CAL).

cessivi, sono stati preparati SI per HIV RNA, HBV DNA, Parvovirus B19 DNA e HAV RNA, come riportato in tabella 3.

Data la loro provenienza "naturale", da campioni di siero o plasma, gli SI hanno una disponibilità limitata, ma possono essere utilizzati per calibrare working reagents, a concentrazione inferiore rispetto agli SI, quali, ad esempio, quelli predisposti e distribuiti dal NIBSC e dall'ISS, sempre costituiti da siero o plasma. Gli standard internazionali servono anche come punto di riferimento per la definizione dei calibratori, cioè dei reagenti che servono a tarare gli strumenti diagnostici in modo che abbiano le stesse performance. Mentre SI (e working reagent) derivano sempre da campioni di siero o plasma contenenti il virus, diluiti in plasma negativo sino alla concentrazione desiderata, i calibratori sono, generalmente, costituiti da sequenze genomiche sintetiche (solitamente plasmidi ricombinanti), a varie concentrazioni a seconda del tipo dello strumento, del metodo di amplificazione e del suo coefficiente di amplifica-

L'uso ripetuto e regolare di controlli interni ed esterni ha, in tempi relativamente brevi, migliorato e stabilizzato le prestazioni dei laboratori di microbiologia nelle applicazioni diagnostiche delle tecniche molecolari.

Questi dati emergono da diversi studi multicentrici condotti per valutare l'efficienza diagnostica dei laboratori partecipanti nell'impiego di tecniche di amplificazione molecolare qualitative e quantitative "classiche" per lo studio di vari marker virali, particolarmente per HCVRNA, per HBVDNA e per l'HIVRNA condotti in vari paesi europei. Anche in Italia, studi promossi dal

Tabella 3. I principali WHO International Standards attualmente in uso

\begin{tabular}{llll} 
•HCV-RNA $(96 / 790)$ & $10^{5} \mathrm{IU} / \mathrm{ml}$ & Saldanha et al & 1999 \\
$\cdot$-HCV-RNA(96/798) & $10^{5} \mathrm{IU} / \mathrm{ml}$ & “ & 2005 \\
•HIV-RNA & $10^{5} \mathrm{IU} / \mathrm{ml}$ & “ & 1999 \\
•HBV-DNA & $10^{6} \mathrm{IU} / \mathrm{ml}$ & “ & 2001 \\
•Parvovirus B19 & $10^{6} \mathrm{IU} / \mathrm{ml}$ & “ & 2002 \\
•HAV-RNA & $10^{5} \mathrm{IU} / \mathrm{ml}$ & “ & 2005 \\
& & & \\
\hline
\end{tabular}
comitato per le Biotecnologie dell'AMCLI (15, 16), effettuati dopo l'introduzione degli standard internazionali, misero in evidenza un'ottima correlazione dei risultati inter-laboratori; infatti le differenze evidenziate tra i vari laboratori rientravano, nella maggior parte dei casi, all'interno di un intervallo di $0.5 \log _{10} \mathrm{UI} / \mathrm{ml}$, insito nella variabilità delle metodiche.

Problemi di standardizzazione della RQ-PCR Con l'introduzione della RQ-PCR, che notoriamente ha un CAL molto più ampio delle tecniche 
di amplificazione classiche, si è visto che, in alcuni casi, particolarmente nella determinazione dell'HCVRNA, i calibratori in uso non riuscivano a correggere le performaces dei vari strumenti; pertanto si notavano grosse differenze tra $i$ dati ottenuti dai vari laboratori.

Infatti, lo studio di Halfon (9) metteva in risalto come su 486 campioni, analizzati con vari sistemi di amplificazione molecolare, tra cui una RQPCR, il $2 \%$ dei valori ottenuti differivano di oltre $2 \log _{10}$. Secondo questo autore, la variabilità nella determinazione della carica virale con l'uso di differenti metodi analitici, tra cui una RQ-PCR, potrebbe dipendere da:

a- le differenze nei range del CAL: la RQ- PCR ha un CAL di circa $2 \log _{10}$ più ampio delle altre metodiche (in particolare rispetto alle metodiche di end-point PCR), ciò potrebbe portare a delle variazioni nella quantizzazione delle concentrazioni più alte $\mathrm{e}$ più basse che gli attuali standard non riescono a correggere. Pertanto l'autore concludeva che il monitoraggio della carica virale in un paziente deve essere effettuato sempre con lo stesso, metodo. Questo era stato evidenziato anche in un nostro studio multicentrico per la standardizzazione delle metodiche molecolari per la ricerca di HCVRNA (15);

b- il rapporto tra copie/ml e UI/ml nei vari metodi in uso, con diversi CAL, forse troppo ampio per uniformare le performances dei diversi sistemi di amplificazione impiegati. Il ricorso alle UI non riesce, quindi, più a correggere queste variazioni.

E probabile che il problema sia da attribuire all'utilizzo dei calibratori piuttosto che agli standard, che rimangono un punto fondamentale in tutto il processo di della standardizzazione dei metodi. E molto indicativo, a questo proposito, lo studio di Watzinger (32). In questo lavoro viene messa in evidenza la diversità dei dati riportati in Letteratura nella determinazione della carica virale in varie infezioni, ottenuti, in molti casi, con l'utilizzo della RQ-PCR; questa discrepanza, secondo gli autori, sarebbe dovuta alla grande diffusione di questa metodica che spesso viene utilizzata senza adeguate informazioni sui sistemi di controllo utilizzati. Forse, ancora più indicativo è il lavoro di Kay-Yin Lai (12), dove viene riportato testualmente che: nonostante il continuo aumento nell'utilizzo della RQ-PCR nella diagnosi e nel monitoraggio delle infezioni virali, non vi sono studi che stabiliscano quali e quanti calibratori è necessari utilizzare e come devono essere utilizzati per un efficiente standardizzazione di questo metodo diagnostico. Diversi altri studi suggeriscono simili conclusioni, tra cui anche quello di Ruiz (23), in cui vengono messe in evidenza le differenze, statisticamente significative, tra due metodi diagnostici che utilizzano la metodologia RQ-PCR nella valutazione della carica virale da virus di Epstein-Barr.

Pertanto il problema si sposta in una ulteriore direzione.

Un problema comune alle metodiche di quantizzazione basate sulla reazione di PCR end-point che non è ancora stato superato dalle tecniche di quantizzazione in real-time, è rappresentato da una diversa efficienza di quantizzazione in rapporto al genotipo virale. Questo problema è oggetto di un importante dibattito soprattutto dopo l'introduzione di metodi di real-time PCR basati sulla tecnologia TaqMan, per virus ad alta variabilità genetica, come HIV e HCV (26). I dati più recenti di confronto tra quantizazzione mediante metodi nonPCR-based, rappresentati per lo più dalla tecnologia di branched-DNA e real-time PCR per HCV fanno emergere per alcuni assemblati TaqMan divergenze di quantizzazione superiori a $1 \mathrm{log}$, rispetto al bDNA, che sembrano essere genotipodipendenti (26). La tecnologia TaqMan, infatti, per la sua costruzione, risulta molto sensibile alla presenza di mis-match nella sequenza amplificata, che sono tanto più frequenti, tanto più elevata è la variabilità gentica virale. Nel tentativo di controllare questo problema, sono stati più recentemente introdotti assemblati real-time per virus ad alta variabilità genetica basati su tecnologie diversa da quella TaqMan (7).

\section{Le curve di calibrazione (Cc) nella RQ-PCR}

Analizzando una serie di studi di autori che possono essere considerati tra $\mathrm{i}$ pionieri di questo argomento $(3,11,14,18)$, emergono le domande fondamentali che bisogna porsi nella costruzione di una curva di calibrazione per la RQ-PCR:

a. quanti calibratori e a quali concentrazioni devono essere utilizzati nell'impostazione di una $\mathrm{Cc}$ ?

b. quando devono essere impostate le Cc rispetto ad ogni singola corsa di amplificazione (run)?

c. come devono essere conservati i calibratori: liofilizzati, congelati? (6)

Nel rispondere a questi quesiti devono essere considerati anche i costi: i calibratori, infatti, hanno un costo non indifferente; secondo lo studio di KaiYin Lai (12), eliminando pochi punti di calibrazione, in un laboratorio con grande mole di lavoro routinario, si può ottenere un sensibile risparmio. Come è noto una $\mathrm{Cc}$ per RQ-PCR viene costruita su due parametri:

a. numero di copie genomiche $/ \mathrm{ml}$ presente in ciascun calibratore (generalmente se ne usano 4), posizionato nell'asse delle $X$; 
b. numero di cicli, per ogni calibratore, in cui viene rilevata la presenza degli ampliconi $(\mathrm{Ct})$, posizionato nell'asse delle Y;

Così impostata la curva mostra due coefficienti fondamentali: lo Slope o coefficiente di inclinazione angolare e l'y-intercept o punto di intersezione della curva sull'asse delle Y. In ogni Cc questi due coefficienti sono espressi in un valore numerico; ogni variazione di questi valori si traduce in una diversa efficienza nelle sedute analitiche. Nella figura I viene mostrata una classica $\mathrm{Cc}$ con le deviazioni standard evidenziate in 50 diverse sedute effettuate con gli stessi calibratori; questi erano costituiti da sequenze genomiche sintetiche conservate a $-20^{\circ} \mathrm{C}$.

Alcuni autori hanno, pertanto, proposto $\mathrm{Cc}$ a diversa impostazione, che potessero risultare più agili da usare e meno costose per un minor consumo dei calibratori. Le $\mathrm{Cc}$ proposte dai vari autori possono essere riassunte come segue:

a) every day run curve: $\mathrm{Cc}$ che viene calcolata per ogni singola seduta. In genere vengono utilizzati 4 calibratori a concentrazioni di 2, $3,4,5 \log _{10}$ copie gnomiche $/ \mathrm{ml}$. Alcuni autori ripetono ogni singolo punto più volte, (12) e adottano il valore medio. Metodo molto dispendioso, che può inserire un errore, quale un'eventuale deviazione standard $(\sigma)$ troppo ampia, relativamente a un basso numero di determinazioni.

b) Stored grand-mean (avenge) curve: la curva viene calcolata, per ogni punto, considerando il valore medio ottenuto da numerose determinazioni per ogni singolo calibratore; successivamente la curva viene messa in memoria ed utilizzata per un periodo di tempo variabile, durante il quale vengono eseguite diverse sedute. L'alto numero di determinazioni stabilizza la $\sigma$.

Generalmente le curve vengono ripetute ad ogni sostituzione della lampada dello strumen-

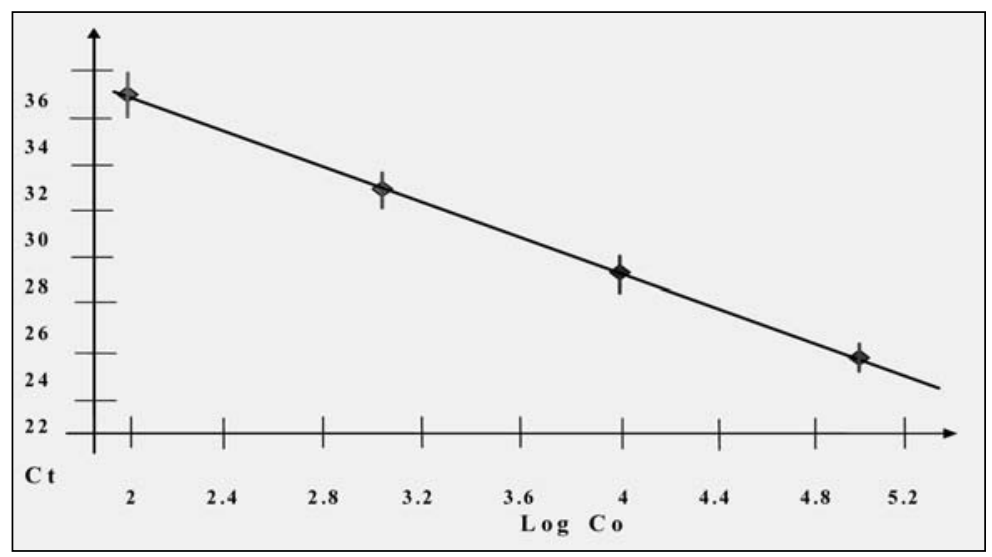

Figura I. Studio della curva di calibrazione per CMV-DNA (ABI Prism 7000) Slope: $-3,47 ; y$ - Intercept: $4 I, 45$ to per evitare al minimo le variazioni di lettura dovute all'efficienza della macchina (18).

c) Daily imported external curve: anche in questo caso la curva viene messa in memoria (avenge curve) e l'impostazione è come la precedente, tuttavia per ogni singola seduta (run) viene calcolato il valore dell'y-intercept con un singolo calibratore, il run control; generalmente viene utilizzato quello a più basse concentrazioni, $2 \log _{10}$ (11). Analizzando i dati riportati dai vari autori in studi comparativi, emerge che i coefficienti di variazione $(\mathrm{CV})$ dei risultati ottenuti sui campioni studiati con le diverse $\mathrm{Cc}$ considerate erano accettabili, tuttavia le $\mathrm{Cc}$ che sembravano dare valori con il più basso $\mathrm{CV}$ erano la every day run cuve e la daily imported external curve (11).

Nel lavoro (12), in cui viene eseguito uno studio comparativo, con l'utilizzo di tutte e tre le curve citate, per la determinazione della carica virale da HBV e da EBV, Kay-Yin Lai considera i valori ottenuti con la every day run curve (each run curve) più accettabili rispetto a quelli ottenuti con le altre due Cc. Inoltre, riporta che l'aggiunta di un run control ad una curva in memoria (avenge curve) non dà ulteriori vantaggi, poiché, secondo i suoi dati, utilizzati per costruire la curva in memoria, risultava che i valori dello Slope variavano più che quelli dell'y-intercept. Viceversa, dai dati ottenuti in nostro studio (Mancini, dati non pubblicati) su 125 pazienti, sottoposti a trapianto d'organo, risultati positivi alla determinazione della carica virale per CMV tramite RQPCR, in cui tutti i campioni di plasma venivano analizzati con tutte e tre le $\mathrm{Cc}$, si sono avute indicazioni diverse. Infatti, i risultati ottenuti con una curva in memoria più l'utilizzo di un run control, a una concentrazione di $2 \log _{10}$, davano dei valori molto simili a quelli ottenuti con una each run cuve, con il CV sempre all'interno di uno scarto pari a $+/-0.2 \log _{10}$; gli stessi campioni valutati con una semplice curva in memoria mostravano uno scarto superiore, + /$0.5 \log _{10}$. Le differenze osservate in questi due studi (Mancini, 12) potrebbero essere dovute alle diverse $\sigma$ relative ai valori utilizzati dai due laboratori nella definizione dei singoli calibratori delle Cc. Infatti, i dati da noi impiegati per designare la curva in memoria (50 per singolo calibratore, contro i circa 60 nel lavoro di Kay-Yin Lai (12)) mostravano delle $\sigma$ molto ristrette che indicavano una bassa variabilità dello Slope.

Da queste considerazioni appare 
chiaro che il problema della standardizzazione delle tecniche diagnostiche che sfruttano metodi di amplificazione molecolare è in piena evoluzione, ma dalla letteratura sull'argomento emerge che due tra i punti più importanti nella standardizzazione sono:

a. la composizione e la conservazione dei calibratori o standard

b. gli studi multicentrici per il controllo di qualità inter-laboratori.

\section{Stabilità dei calibratori}

Sono molte gli studi in cui vengono proposti vari approcci di tipo statistico/matematico (14), per ridurre al minimo le variabili che possono alterare i dati ottenuti da una tecnica di amplificazione molecolare. Tuttavia, è ormai accertato che le performances dei diversi lotti di reagenti e una certa instabilità dei calibratori, durante la conservazione, sono tra i principali artefici delle differenze nell'efficienza di una RQ-PCR $(6,11,14)$.

La maggioranza degli autori ritiene che i calibratori/standard debbano essere mantenuti liofilizzati e congelati (11); su quest'aspetto molto influisce la stabilità dei calibratori. Infatti noi conserviamo i nostri calibratori a $-20^{\circ} \mathrm{C}$ mantenendoli i ghiaccio durante l'uso, e le nostre $\sigma$, come prima riportato (Mancini, dati non pubblicati) sono molto ristrette.

L'utilizzo di un run control, particolarmente quando si usano Cc in memoria (avenge) o le imported external cuves, oltre a permettere una più precisa valutazione del valore dell' $y$-intrcept, può dare utili informazioni relative al funzionamento dei controlli. I laboratori dovrebbero conoscere il valore del range di accettabilità, o della $\sigma$, dei valori dei calibratori utilizzati, per poter correttamente valutare $\mathrm{i}$ risultati ottenuti e provvedere di conseguenza.

\section{Controlli esterni di qualità (QCE)}

I QCE sono attualmente entrati nella prassi routinaria di tutti i laboratori diagnostici. Tuttavia nei laboratori ove si utilizzano metodologie di amplificazione molecolare, la partecipazione a studi multicentrici per il controllo di qualità inter-laboratori è di fondamentale importanza.

Per questo, il Comitato di Studio per la Biologia Molecolare (CoSBioM) dell'AMCLI, già dall'inizio degli anni 2000, ha organizzato degli studi multicentrici per valutare il livello di standardizzazione dei laboratori italiani nella diagnostica microbiologica molecolare. Un primo studio era rivolto ai laboratori che effettuavano diagnosi di infezione da HCV con saggi qualitativi (16); successivamente è stato impostato un secondo studio per la valutazione della standardizzazione delle tecniche diagnostiche di tipo quantitativo, sempre per HCV RNA (15). L'indagine policentrica era sempre preceduta da attente valutazioni del Comitato su quali campioni-standard dovevano essere considerati idonei per un QC e gli obiettivi da raggiungere. In primo luogo, gli standard dovevano avere tutte le caratteristiche di un normale campione clinico e dovevano arrivare ai laboratori integri nella loro composizione. Inoltre il pannello di campioni inviati a ciascun laboratorio doveva essere a concentrazioni genomiche limite, relativamente alla sensibilità e alla specificità degli strumenti analizzati. Infatti, in uno studio per il QC è conveniente raggruppare i laboratori partecipanti in base alle metodologie analitiche applicate, e quindi in base al coefficiente di amplificazione lineare (CAL) relativo ai metodi in uso.

Oltre a valutare la sensibilità e la specificità ottenuta da ogni laboratorio, è importante valutare anche la ripetitività del dato; pertanto nella composizione di un pannello analitico devono esserci campioni positivi, a varie concentrazioni (oltre a quelle limite), campioni negativi e anche alcuni campioni con la stessa concentrazione genomica, per una più completa verifica dell'efficienza di un laboratorio. Attualmente, come riportato nella tabella 4, a livello europeo il più autorevole ente che si occupa di controlli di qualità per i saggi molecolari è il Quality Control for Molecular Diagnostic (QCMD), supportato dalla Commissione Europea e riconosciuto dalla European Society (E.S.) of Clinical Virology e dalla E.S. of Clinical Microbiology and Infectious Diseases. Il QMCD fornisce controlli di qualità e organizza programmi per la valutazione esterna della qualità a livello Europeo. Questo Ente ha cominciato la sua attività già dal 1998 con uno studio sugli Enterovirus

Tabella 4. Enti Internazionali per la Standardizzazzione nella Diagnostica Molecolare e programmi VEQ nell'ambito della diagnostica molecolare in microbiologia e virologia

$\underline{\text { WHO Export Committee on Biological Standardization }}$

a) Definizione degli Standards

UK National External Quality Assessment Scheme (NEQAS)

a)Impostazione dei programmi per il QC extra-laboratorio

Quality Control for Molecular Diagnostic-QCMD

a) Definizione dei Controlli di Qualità

b) Impostazione dei programmi per il QC extra-laboratorio 
e, in questi anni, si è occupato della standardizzazione delle tecniche molecolari usate per la diagnosi di molte infezioni, quali, in particolare, quelle da virus dell'epatite $\mathrm{B}$ e $\mathrm{C}$ (valutazione qualitativa e quantitativa degli acidi nucleici e determinazione del genotipo virale), Chlamydia, CMV, EBV, HSV, VZV e M. tuberculosis. Dall'analisi dei dati riportati dal QCMD, si può osservare, che le performance dei laboratori migliorano continuamente con la partecipazione a questi QC. Sono attualmente in fase di studio programma di esterni di controllo di qualità su polyomavirus JC e BK, virus dell'influenza A e B, estendendo in tal modo anche il range di materiale biologico dal plasma ad altri materiali come quelli respiratori.

Un altro importante organismo scientifico che propone programmi di verifica esterna di qualità con controlli calibrati secondo WHO, è rappresentato dal UK National External Quality Assessment Scheme (NEQAS) attivo da molti anni in ambito microbiologico, con vasta adesione da parte dei laboratori italiani. NEQAS ha da tempo esteso il ricco ventaglio di proposte di VEQ ai virus epatitici B e C e HIV, sia per la valutazione qualitativa e quantitativa degli acidi nucleici che per la determinazione del genotipo virale di $\mathrm{HCV}$ e la farmacoresistenza di HIV. Lo stesso schema prevede anche l'invio di materiali biologici diversi da plasma, per altre indagini virologiche sempre di tipo molecolare, come liquor cefalorachidiano per $\mathrm{i}$ test molecolari sui virus erpetici (HSV 1 e 2, VZV, CMV e EBV) o altri materiali per indagini molecolari in ambito batteriologico, micologico, parassitologico e micobatteriologico. Più recentemente, il programma si è esteso a CMV.

\section{Alcune delle standardizzazioni da affrontare: HPV e polyomavirus BK (BKV)}

I sistemi attualmente disponibili per la diagnosi di infezione da HPV ad alto rischio oncogeno si basano fondamentalmente sull'ibridazione dell'acido nucleico bersaglio con sonde marcate e amplificazione del segnale delle sonde (Hybrid Capture, HC) oppure su saggi di amplificazione genica con successiva genotipizzazione mediante sonde marcate. Il sistema HC, che utilizza un pool di sonde per la ricerca di 13 genotipi di HPV prevalenti ad alto rischio, è l'unico sistema attualmente approvato dall'FDA. Altri test che prevedono l'amplificazione della sequenza genica bersaglio e successiva genotipizzazione mediante sonde specifiche sono comunque disponibili commercialmente e portano il marchio CE. I diversi saggi di PCR commerciali e "in house", descritti in Letteratura, amplificano diverse sequenze del genoma di HPV (prevalentemente L1, E6 e E2) e nell'ambito dei diversi amplificati è possibile poi tipizzare un diverso numero di genotipi $(1,8,30)$. I principali problemi di standardizzazione sono relativi all'efficienza con cui sono identificati i diversi genotipi nell'ambito di uno stesso amplificato, la presenza di infezioni sostenute da più ceppi di HPV, il numero di genotipi ad alto rischio che possono essere identificati nell'ambito di uno stesso amplificato e l'assenza di cross reattività nell'identificazione di diversi genotipi nell'ambito dello stesso amplificato.

È auspicabile un controllo di qualità che permetta la normalizzazione dei diversi saggi disponibili, anche al fine di monitorare l'impatto della vaccinazione $(19,20)$. Purtroppo, non ci sono fonti naturali di materiale biologico per preparare dei reagenti standard tipo-specifici. Generalmente, i campioni biologici contengono poche copie di genomi di HPV e nello stesso campione sono presenti genotipi diversi. Inoltre, non sono disponibili, attualmente, metodi colturali adatti per produrre standard di HPV in sistemi cellulari in vitro. Pertanto, i reagenti utilizzabili come controlli sono rappresentati da plasmidi contenenti le intere sequenze genomiche di HPV di genotipi diversi. In un primo studio multicentrico internazionale promosso dall'WHO per la ricerca di HPV (21) è stato utilizzato un panello di 8 plasmidi contenenti l'intera sequenza di DNA di HPV 6, 16, 18, $31,33,35,45$, e 52 , rispettivamente, ad una concentrazione di circa $10^{11}$ genomi $/ \mathrm{ml}$. Per simulare, per quanto possibile, le condizioni dei campioni naturali, il DNA plasmidico veniva diluito nel DNA genomico di cellule epiteliali C33A, una linea proveniente da un carcinoma cervicale umano, HPV negativo.

La necessità di diagnosticare una infezione da polyomavirus BK è emersa negli ultimi anni in relazione, soprattutto, all'aumento della nefropatia polyomavirus associata (PVAN) nei trapiantati renali e alla necessità di fare una diagnosi differenziale in funzione delle diverse possibilità terapeutiche. Essendo un virus che causa infezioni latenti, le riattivazioni, soprattutto nei pazienti immunodepressi, sono frequenti, ma solitamente le manifestazioni cliniche sono accompagnate da una carica virale elevata nelle urine e anche nel siero. Da qui l'esigenza ai fini diagnostici di disporre di saggi di tipo quantitativo. Diversi saggi di PCR "in house" sono stati descritti in letteratura $(5,22,28)$, alcuni saggi di real time PCR sono attualmente disponibili commercialmente, almeno 2 dei quali con marchi CE. La standardizzazione dei metodi quantitativi è particolarmente necessaria in quanto sono proposti in Letteratura dei livelli di carica associati allo sviluppo delle 
manifestazioni cliniche e quindi all'esigenza di interventi terapeutici.

\section{CONCLUSIONI}

I dati riportati complessivamente suggeriscono alcune considerazioni conclusive:

- la standardizzazione è ancora carente in diversi settori della diagnostica microbiologica molecolare, come illustrato degli ultimi due esempi citati;

- l'evoluzione delle metodiche molecolari può richiedere una verifica e un adeguamento dei controlli da utilizzare nelle reazioni;

- sono ancora pochi gli SI o anche i controlli internazionalmente riconosciuti

- attualmente il QCMD e il NEQAS sono i principali enti che a livello europeo promuovono la standardizzazione delle tecniche molecolari, tramite programmi di verifica esterna della qualità.

Tuttavia i laboratori italiani che hanno partecipato a questi studi, benché siano aumentati negli ultimi anni, non sono stati molti. Questa considerazione e il fatto che da questi studi non si ha una chiara visione della realtà locale, suggeriscono l'esigenza di un organismo che si occupi della programmazione e dell'organizzazione di QC in ambito nazionale, anche se, auspicabilmente, in stretta collaborazione con il QCMD e il NEQAS.

\section{BIBLIOGRAFIA}

1. Bonvicini F, Venturoli S, Ambretti S, et al. Presence and type of oncogenic human papillomavirus in classic and in differentiated vulvar intraepithelial neoplasia and keratinizing vulvar squamous cell carcinoma. J Med Virol 2005; 77: 102-6.

2. Buchbinder A, Josephs SF, Ablashi D, et al. Polymerase chain reaction amplification and in situ hybridization for the detection of human B-lymphotropic virus. J. Virol. Methods 1988; 21: 191-7.

3. Burns MJ, Nixon GJ, Foy CA, Harris N. Standardisation of data from real-time quantitative PCR methods evaluation of outlier and comparision of calibration curves. BMC Biotechnologies 2005; 5: 31 .

4. Compton J. Nucleic acid sequence-based amplification. Nature 1991; 350: 91-2.

5. Elfaitouri A, Hammarin AL, Blomberg J. Quantitative real-time PCR assay for detection of human polyomavirus infection. J Virol Methods 2006; 135: 207 13.

6. Farriol M, Orta X. Influence of reagent formulation on mRNA quantification by RT-PCR using imported external standard curves. Acta Biochim Pol 2005; 52: 845-8.

7. Gardner SN, Kuczmarski TA, Vitalis EA, Slezak TR. Limitations of TaqMan PCR for detecting divergent viral pathogens illustrated by hepatitis $\mathrm{A}, \mathrm{B}, \mathrm{C}$, and $\mathrm{E}$ viruses and human immunodeficiency virus. J Clin Microbiol 2003; 41: 2417-27.

8. Gravitt PE, Peyton CL, Apple RJ, Wheeler CM. Genotyping of 27 human papllomavirus types by using L1 consensus PCR products by a single -hybridization, reverse line blot detection method. J Clin Microbiol 1998; 36: 3020-7.

9. Halfon $P$, Pènaranda $G$, Bourlière $M$, Khiri $H$, Masseyeff MF, Ouzan D. Assessment of early virological response to antiviral therapy by comparing four assays for HCV RNA quantitation using the International unit standard: implication for clinical management of patients with chronic hepatitis $\mathrm{C}$ virus infection. J Med Virol 2006; 78: 208-15.

10. Hill CS. Molecular diagnostic testing for infectious diseases using TMA technology. Expert Rev Mol Diagn 2001; 42: 3041-5.

11. Hymas W, Stevenson J, Taggart EW, Hillyard D. Use of lyophilized standards for the calibration of a newly developed real time PCR assay for human herpes type six (HH6) variant A and B. J Virol Methods 2005; 128: 143-50.

12. Lai KK, Cook L, Krantz EM, Corey L, Jerome KR. Calibration curves for real-time PCR. Clin Chem; 51: 1132-6.

13. Landegren U. Ligation-based DNA diagnostics. Bioessays. 1993; 15: 761-5.

14. Larionov A, Krause A, Miller W. A standard curve based method for relative real time PCR data processing. BMC Bioinformatics 2005; 6: 62.

15. Mancini C, Pisani G, Azzi A, Zerbini ML, Gentili G, Mario Bisso G, MS participants. Inter-laboratory comparision of qualitative and quantitative detection of hepatitis $\mathrm{C}(\mathrm{HCV})$ virus RNA in diagnostic virology; a multicentre study (MS) in Italy. J Clin Virol; 30: 313-9.

16. Mancini C, Zerbini M, Azzi A, Piunno M. Multicentre Italian Study Group (MISG) for standardisation of hepatitis C virus (HCV) PCR. J Clin Virol 2003; 27 (7): 83-9.

17. Murakawa GJ, Zaia JA, Spallone PA, et al. Direct detection of HIV-1 RNA from AIDS and ARC patient samples. DNA 1988; 7: 287-95.

18. Ovestebo R, Haug KB, Lande K, Kierulf P. PCRbased calibration curves for studies of quantitative gene expression in human monocytes: development and evaluation. Clin Chem 2003; 49: 425-32.

19. Pagliusi SR, Dillner J, Pawlita M, Quint WG, Wheeler CM, Ferguson M. Charter 23: International Standard reagents for harmonization of HPV serology and DNA assay-an update. Vaccine 24S3 (2006); S3/193$\mathrm{S} 3 / 200$.

20. Pagliusi SR, Garland SM. International Standard reagents for HPV detection. Dis Markers 2007; 23: 283-96.

21. Quint WG, Pagliusi SR, Lelie N, de Villiers EM, Wheeler CM. World Health Organization Human Papillomavirus DNA International Collaborative Study Group. Results of the first World Health Organization international collaborative study of detection of human papillomavirus DNA. J Clin Microbiol 2006; 44: 571-9.

22. Randhawa P, Ho A, Shapiro R, Vats A, et al. Correlates of quantitative measurement of BK polyomavirus (BKV) DNA with clinical course of BKV infection in renal transplant patients. J Clin Microbiol 2004; 42: 1176-80.

23. Ruiz G, Pena P, de Ory F, Echevarria JE. Comparision of commercial real-time PCR assays for quantification of Epstein-Barr virus DNA. J Clin Microbiol; 43: 2053-7.

24. Saiki RK, Scharf S, Faloona F, et al. Enzymatic amplification of beta-globin genomic sequences and restriction site analysis for diagnosis of sickle cell 
anemia. Science 1985; 230: 1350-4.

25. Saldanha J, Lelie N, Heath A. WHO Collaborative Study Group. Establishment of the first international standard for nucleic acid amplification technology (NAT)assay for HCV-RNA. Vox Sang 1999; 76: 14958.

26. Sarrazin C, Gartner BC, Sizmann D, et al. Comparison of conventional PCR with real-time PCR and branched DNA-based assays for hepatitis $\mathrm{C}$ virus RNA quantification and clinical significance for genotypes 1 to 5. J Clin Microbiol 2006; 44: 729-37.

27. Schiffman MH, Kiviat NB, Burk RD, et al. Accuracy and interlaboratory reliability of human papillomavirus DNA testing by hybrid capture. J Clin Microbiol 1995; 33: 545-50.

28. Sehbani L, Kabamba-Mukadi B, Vandenbroucke AT, Bodeus M, Goubau P. Specific and quantitative detection of human polyomaviruses $\mathrm{BKV}$ and $\mathrm{JCV}$ by LightCycler real-time PCR. J Clin Virol 2006; 36: 159-62.
29. Urdea MS. Branched DNA signal amplification. Biotechnology (N Y). 1994; 12: 926-8.

30. vanDoorn LJ, Kleter B, Quint WG. Molecula detection and genotyping of human papillomavirus. Exp Rev Mol Diagn 2001; 1: 394-402.

31. Walker GT, Fraiser MS, Schram JL, Little MC, Nadeau JG, Malinowski D. Strand displacement amplification - an isothermal, in vitro DNA amplification technique. Nucleic Acids Res 1992; 20: 1691-6.

32. Watzinger F, Ebner K, Lion T. Detection and monitoring of virus infection by real-time PCR. Molecular Aspects of Medicine 2006; 27: 254-98.

33. Yang S, Rothman RE. PCR-based diagnostics for infectious diseases: uses, limitations and future applications in acute-care settings. Lancet infect dis 2004; 4: $337-48$

34. Zaaijer HL, Cuypers HT, Reesink HM, Winkel IN, Getrken G, Lelie PN. Reliability of polymerase chain reaction of detection of hepatitis $\mathrm{C}$ virus. Lancet 1993; 341: 722-4. 